\title{
An Extended Linear Quadratic Regulator and its Application for Control of Power System Dynamics
}

\author{
Abhinav K. Singh*, Member, IEEE, and Bikash C. Pal ${ }^{\dagger}$, Fellow, IEEE \\ Control and Power Research Group \\ Department of Electrical and Electronic Engineering \\ Imperial College London, London, United Kingdom \\ Email: *a.singh11@imperial.ac.uk, †b.pal@imperial.ac.uk
}

\begin{abstract}
This paper presents a generalized solution to the problem of optimal control of systems having an extra set of exogenous inputs besides control inputs. The solution is derived in the framework of linear quadratic control and it is termed 'extended linear quadratic regulator (ELQR)'. The ELQR is applied for control of unstable or poorly damped oscillatory dynamics occurring in a power system and is shown to be significantly more cost effective than the classical power system stabilizer (PSS) based approach.

Index Terms - optimal control, extended linear quadratic regulator, exogenous input, external disturbance, pseudo-input, power system dynamics, small signal stability, power system stabilizer
\end{abstract}

\section{NOMENCLATURE}

$\mathbf{0}_{m \times n} \quad$ a matrix of zeroes of size $(m \times n)$

$\boldsymbol{\Gamma} \quad$ the function of quadratic costs without considering $\boldsymbol{u}^{\prime}$

$\Gamma^{\prime} \quad$ the quadratic cost function considering both $\boldsymbol{u}$ and $\boldsymbol{u}^{\prime}$

$\boldsymbol{A}$ the matrix corresponding to states

$\boldsymbol{B}$ the matrix corresponding to $\boldsymbol{u}$

$\boldsymbol{E}$ the matrix corresponding to $\boldsymbol{u}^{\prime}$

$G$ the gain corresponding to state-feedback

$\boldsymbol{I}_{m} \quad \mathrm{a}(m \times m)$ identity matrix

$\boldsymbol{K}$ the feedback gain corresponding to $\boldsymbol{u}^{\prime}$

$\boldsymbol{K}^{\prime}$ the supplementary feedback in ELQR

$\boldsymbol{L}$ the matrix corresponding to $\boldsymbol{K}$ in ELQR

$\boldsymbol{L}^{\prime} \quad$ the matrix corresponding to $\boldsymbol{K}^{\prime}$ in ELQR

$\boldsymbol{P}$ algebraic Riccati equation's positive definite solution

$\boldsymbol{Q}$ the cost matrix of $\boldsymbol{x}$

$\boldsymbol{R}$ the cost matrix of $\boldsymbol{u}$

$\boldsymbol{S}$ the cost matrix of $\boldsymbol{u}^{\prime}$

$\boldsymbol{u}$ the control inputs' vector

$\boldsymbol{u}^{\prime}$ the exogenous inputs' vector

$\boldsymbol{x}$ the states' vector

$k \quad$ the $k^{t h}$ time sample

$M \quad$ the final time for reaching steady state

$T \quad$ the symbol for transpose

$T_{0} \quad$ the system's sampling period in $\mathrm{s}$

\section{INTRODUCTION}

$\mathbf{T}$ HE branch of control-systems theory that studies costminimizing operation of a dynamic system by placing

Grants EESC-P55251 and EP/K036173/1 from EPSRC, U.K., support this work. constraints on control effort is known as optimal control. A specific case of optimal control is the linear quadratic (LQ) problem, in which the system dynamics are described by linear and time invariant (LTI) differential equations and the associated cost (which needs to be optimized) is a quadratic function of system state-deviations and control-efforts [1]. It was shown by R. E. Kalman that the solution to the LQ problem is a state feedback controller with a constant gain and the solution was termed 'linear quadratic regulator (LQR)' [2].

The inputs which are considered in the LQ problem are control inputs, which means that each input applied to the system can be fully altered by the controller. But, this is not true for a general system, and some of the inputs may also be external disturbances which can neither be altered nor be disabled. These inputs are also known as exogenous inputs. Some examples of systems with external disturbances in control literature can be found in [3], [4] and [5]. A recent example in power system literature can also be found in [6], wherein external disturbances are called 'pseudo-inputs'.

The optimal control problem for systems with exogenous inputs can be found in studies such as [7]-[9]. In these studies, the exogenous input is accommodated in the LQR solution by finding a control input which minimizes the effect of the exogenous input on the system dynamics. But, this does not optimize the net costs corresponding to controlefforts and deviations in system states. Thus, the technique of accommodating the exogenous input in the LQR solution fails to achieve the chief objective of optimal control.

A new solution to the problem of optimal control of systems with exogenous inputs has been proposed in this paper, and the solution not only guarantees optimal accommodation of the external disturbance, but also optimizes the net quadratic costs corresponding to control-efforts and deviations in states. The solution is valid for any sequence of external disturbances. The practical applicability of the method is demonstrated for power systems, and the proposed method is employed for decentralized control for ensuring small-signal stability of a large-scale power system model.

Rest of the paper is organized as follows. Section II briefly explains the classical LQR solution, while Section III describes the proposed solution. The solution is demonstrated on a model of a power system in Section IV. Section V is the 
concluding section.

\section{LINEAR QUADRATIC REGULATOR}

An open-loop LTI system without any external disturbance can be described by the following discrete-time equation.

$$
\boldsymbol{x}_{k+1}=\boldsymbol{A} \boldsymbol{x}_{k}+\boldsymbol{B} \boldsymbol{u}_{k}
$$

The quadratic cost for the above system for $M+1$ samples can be written as:

$$
\boldsymbol{\Gamma}=\sum_{k=0}^{M}\left[\boldsymbol{x}_{k}^{T} \boldsymbol{Q} \boldsymbol{x}_{k}+\boldsymbol{u}_{k}^{T} \boldsymbol{R} \boldsymbol{u}_{k}\right] \text { where } \boldsymbol{Q} \geq 0, \boldsymbol{R}>0
$$

If $\boldsymbol{\Gamma}$ is minimized with respect to (w.r.t.) $\boldsymbol{u}_{k}$ then the LQR solution is obtained as follows.

$$
\begin{aligned}
& \boldsymbol{u}_{k}=-\boldsymbol{G}_{k} \boldsymbol{x}_{k}, \quad k=0,1, \ldots,(M-1), \boldsymbol{u}_{M}=\mathbf{0} ; \text { where }, \\
& \boldsymbol{G}_{k-1}=\left(\boldsymbol{R}+\boldsymbol{B}^{T} \boldsymbol{P}_{k} \boldsymbol{B}\right)^{-1} \boldsymbol{B}^{T} \boldsymbol{P}_{k} \boldsymbol{A}, \boldsymbol{P}_{M}=\boldsymbol{Q}, \text { and } \\
& \boldsymbol{P}_{k-1}=\boldsymbol{Q}+\boldsymbol{A}^{T}\left[\boldsymbol{P}_{k}-\boldsymbol{P}_{k} \boldsymbol{B}\left(\boldsymbol{R}+\boldsymbol{B}^{T} \boldsymbol{P}_{k} \boldsymbol{B}\right)^{-1} \boldsymbol{B}^{T} \boldsymbol{P}_{k}\right] \boldsymbol{A}
\end{aligned}
$$

The optimal control policy obtained above is finite horizon if $M$ is finite; otherwise it is called infinite horizon policy. $\boldsymbol{P}_{k}$ and $\boldsymbol{G}_{k}$ are bounded in the case of infinite horizon and a solution exists for them if and only if $(\boldsymbol{A}, \boldsymbol{B})$ is shown to be stabilizable. The following algebraic Riccati equation (ARE) needs to be solved to obtain the steady-state solution.

$$
\begin{gathered}
\boldsymbol{P}=\boldsymbol{Q}+\boldsymbol{A}^{T}\left[\boldsymbol{P}-\boldsymbol{P} \boldsymbol{B}\left(\boldsymbol{R}+\boldsymbol{B}^{T} \boldsymbol{P} \boldsymbol{B}\right)^{-1} \boldsymbol{B}^{T} \boldsymbol{P}\right] \boldsymbol{A} \\
\boldsymbol{G}=\left(\boldsymbol{R}+\boldsymbol{B}^{T} \boldsymbol{P} \boldsymbol{B}\right)^{-1} \boldsymbol{B}^{T} \boldsymbol{P} \boldsymbol{A}
\end{gathered}
$$

\section{EXTENDED LINEAR QUADRATIC REGULATOR}

An open-loop LTI system with external disturbances can be described by the following discrete-time equation.

$$
\boldsymbol{x}_{k+1}=\boldsymbol{A} \boldsymbol{x}_{k}+\boldsymbol{B} \boldsymbol{u}_{k}+\boldsymbol{E} \boldsymbol{u}_{k}^{\prime}
$$

The following equation states the above system's quadraticcosts for $M+1$ samples.

$$
\begin{array}{r}
\boldsymbol{\Gamma}^{\prime}=\sum_{k=0}^{M}\left[\boldsymbol{x}_{k}^{T} \boldsymbol{Q} \boldsymbol{x}_{k}+\boldsymbol{u}_{k}^{T} \boldsymbol{R} \boldsymbol{u}_{k}+\boldsymbol{u}_{k}^{\prime T} \boldsymbol{S} \boldsymbol{u}_{k}^{\prime}\right], \text { where, } \\
\boldsymbol{Q} \geq 0, \boldsymbol{R}>0, \boldsymbol{S} \geq 0 ;
\end{array}
$$

The optimal control policy for (8) can be found by minimizing $\Gamma^{\prime}$ in (9) w.r.t. $\boldsymbol{u}_{k}$. The minimization leads to the following optimal control law.

Theorem 1. For an open-loop linear time invariant system having external disturbances (described by (8)), such that $\boldsymbol{u}_{k}^{\prime}=\mathbf{0} \forall k \geq M, \boldsymbol{A}$ is non-singular and $\boldsymbol{u}_{k}^{\prime}$ is finite and measurable, the optimal control law for $0 \leq k<M$ is described by (10)-(12) and for $k \geq M$ it is $\boldsymbol{u}_{k}=\mathbf{0}$.

$$
\begin{gathered}
\boldsymbol{u}_{k}=-\left(\boldsymbol{G}_{k} \boldsymbol{x}_{k}+\boldsymbol{K}_{k} \boldsymbol{u}_{k}^{\prime}+\boldsymbol{K}_{k}^{\prime}\right) \\
\boldsymbol{K}_{k}=\boldsymbol{G}_{k}\left(\boldsymbol{P}_{k}-\boldsymbol{Q}\right)^{-1} \boldsymbol{L}_{k}, \boldsymbol{K}_{k}^{\prime}=\boldsymbol{G}_{k}\left(\boldsymbol{P}_{k}-\boldsymbol{Q}\right)^{-1} \boldsymbol{L}_{k}^{\prime} \\
\boldsymbol{L}_{M}=\mathbf{0}, \boldsymbol{L}_{M}^{\prime}=\mathbf{0}, \boldsymbol{L}_{k}=\left(\boldsymbol{A}-\boldsymbol{B} \boldsymbol{G}_{k}\right)^{T}\left(\boldsymbol{P}_{k+1} \boldsymbol{E}+\boldsymbol{L}_{k+1}\right), \\
\boldsymbol{L}_{k}^{\prime}=\left(\boldsymbol{A}-\boldsymbol{B} \boldsymbol{G}_{k}\right)^{T}\left(\boldsymbol{L}_{k+1}\left(\boldsymbol{u}_{k+1}^{\prime}-\boldsymbol{u}_{k}^{\prime}\right)+\boldsymbol{L}_{k+1}^{\prime}\right)
\end{gathered}
$$

$\boldsymbol{G}_{k}$ and $\boldsymbol{P}_{k}$ are given by (4)-(5), $\boldsymbol{u}_{k} \in \mathbb{R}^{l \times 1}, \boldsymbol{x}_{k} \in \mathbb{R}^{m \times 1}$, $\boldsymbol{u}_{k}^{\prime} \in \mathbb{R}^{n \times 1}, \boldsymbol{G}_{k} \in \mathbb{R}^{l \times m}, \boldsymbol{K}_{k} \in \mathbb{R}^{l \times n}, \boldsymbol{K}_{k}^{\prime} \in \mathbb{R}^{l \times 1}, \boldsymbol{P}_{k} \in$ $\mathbb{R}^{m \times m}, \boldsymbol{Q} \in \mathbb{R}^{m \times m}, \boldsymbol{L}_{k} \in \mathbb{R}^{m \times n}, \boldsymbol{L}_{k}^{\prime} \in \mathbb{R}^{m \times 1}, \boldsymbol{A} \in \mathbb{R}^{m \times m}$, $\boldsymbol{B} \in \mathbb{R}^{m \times l}$ and $\boldsymbol{E} \in \mathbb{R}^{m \times n}$.

Proof. The system described by (8) needs to be modified slightly in order to derive Theorem 1 . The modification is to append a constant external disturbance to $\boldsymbol{u}_{k}^{\prime}$ as follows.

$$
\begin{aligned}
& \boldsymbol{x}_{k+1}=\boldsymbol{A} \boldsymbol{x}_{k}+\boldsymbol{B} \boldsymbol{u}_{k}+\boldsymbol{F} \boldsymbol{w}_{k} ; \\
& \text { where, } \boldsymbol{w}_{k}=\left[\begin{array}{c}
\boldsymbol{u}_{k}^{\prime} \\
1
\end{array}\right], \boldsymbol{F}=\left[\begin{array}{ll}
\boldsymbol{E} & \mathbf{0}_{m \times 1}
\end{array}\right] \\
& \boldsymbol{w}_{k}=\boldsymbol{J}_{k} \boldsymbol{w}_{k-1}, \text { where, } \boldsymbol{J}_{k}=\left[\begin{array}{cc}
\boldsymbol{I}_{n} & \boldsymbol{u}_{k}^{\prime}-\boldsymbol{u}_{k-1}^{\prime} \\
\mathbf{0}_{1 \times n} & 1
\end{array}\right] ; \\
& \boldsymbol{F} \boldsymbol{w}_{k}=\left[\begin{array}{ll}
\boldsymbol{E} & \mathbf{0}_{m \times 1}
\end{array}\right]\left[\begin{array}{c}
\boldsymbol{u}_{k}^{\prime} \\
1
\end{array}\right]=\boldsymbol{E} \boldsymbol{u}_{k}^{\prime}+\mathbf{0}_{m \times 1}=\boldsymbol{E} \boldsymbol{u}_{k}^{\prime}
\end{aligned}
$$

As mentioned earlier, $\boldsymbol{x}_{k} \in \mathbb{R}^{m \times 1}$ and $\boldsymbol{u}_{k}^{\prime} \in \mathbb{R}^{n \times 1}$. The equalities in (15) shows that appending a constant external disturbance, 1, does not effect the original system's dynamics. This is required to find an iterative policy for optimal control. It is not possible to express $\boldsymbol{u}_{k}^{\prime}$ in terms of $\boldsymbol{u}_{k-1}^{\prime}$ unless a new external disturbance $\boldsymbol{w}_{k}$ is defined using (13). By doing this, it is possible to express $\boldsymbol{w}_{k}$ in terms of $\boldsymbol{w}_{k-1}$ using (14). The net quadratic-cost for $M+1$ samples for the new system (described by (13)) is given as follows.

$$
\boldsymbol{\Gamma}^{\prime}=\sum_{k=0}^{M}\left[\boldsymbol{x}_{k}^{T} \boldsymbol{Q} \boldsymbol{x}_{k}+\boldsymbol{u}_{k}^{T} \boldsymbol{R} \boldsymbol{u}_{k}+\boldsymbol{w}_{k}^{T} \boldsymbol{U} \boldsymbol{w}_{k}\right]
$$

where, $\boldsymbol{U}=\left[\begin{array}{cc}\boldsymbol{S} & \boldsymbol{0}_{n \times 1} \\ \mathbf{0}_{1 \times n} & 0\end{array}\right], \boldsymbol{Q} \geq 0, \boldsymbol{R}>0, \boldsymbol{S} \geq 0$

$$
\boldsymbol{w}_{k}^{T} \boldsymbol{U} \boldsymbol{w}_{k}=\left[\begin{array}{ll}
\boldsymbol{u}_{k}^{\prime T} & 1
\end{array}\right]\left[\begin{array}{cc}
\boldsymbol{S} & \mathbf{0}_{n \times 1} \\
\mathbf{0}_{1 \times n} & 0
\end{array}\right]\left[\begin{array}{c}
\boldsymbol{u}_{k}^{\prime} \\
1
\end{array}\right]=\boldsymbol{u}_{k}^{\prime T} \boldsymbol{S} \boldsymbol{u}_{k}^{\prime}
$$

The equations (18) and (17) show that the constant external disturbance 1 in $\boldsymbol{w}_{k}$ does not have any associated cost. Thus, the two systems described by (16) and (9), respectively, have identical quadratic costs.

Since $\boldsymbol{u}_{k}^{\prime}=\mathbf{0} \forall k \geq M$ and the steady state of the system, $\boldsymbol{x}_{M}$, is reached at $k=M$, therefore the optimal control input is $\boldsymbol{u}_{k}=\mathbf{0} \forall k \geq M$. The cost for $k=M$ is, thus, optimal and is given by $\boldsymbol{\Gamma}_{M}^{\prime o p t}=\boldsymbol{x}_{M}^{T} \boldsymbol{Q} \boldsymbol{x}_{M}=\boldsymbol{x}_{M}^{T} \boldsymbol{P}_{M} \boldsymbol{x}_{M}$. The net cost for $k=M-1$ and $k=M$, such that the cost for $k=M$ is optimal, is given as follows.

$$
\begin{aligned}
\boldsymbol{\Gamma}_{M-1}^{\prime}=\boldsymbol{x}_{M-1}^{T} \boldsymbol{Q} \boldsymbol{x}_{M-1}+ & \boldsymbol{u}_{M-1}^{T} \boldsymbol{R} \boldsymbol{u}_{M-1} \\
& +\boldsymbol{w}_{M-1}^{T} \boldsymbol{U} \boldsymbol{w}_{M-1}+\boldsymbol{\Gamma}_{M}^{\prime o p t}
\end{aligned}
$$

Substituting $\boldsymbol{\Gamma}_{M}^{o p t}=\boldsymbol{x}_{M}^{T} \boldsymbol{P}_{M} \boldsymbol{x}_{M}$ and $\boldsymbol{x}_{M}=\boldsymbol{A} \boldsymbol{x}_{M-1}+$ $\boldsymbol{B} \boldsymbol{u}_{M-1}+\boldsymbol{F} \boldsymbol{w}_{M-1}$ in (19), gives the following expression.

$$
\begin{array}{r}
\boldsymbol{\Gamma}_{M-1}^{\prime}=\boldsymbol{x}_{M-1}^{T} \boldsymbol{Q} \boldsymbol{x}_{M-1}+\boldsymbol{u}_{M-1}^{T} \boldsymbol{R} \boldsymbol{u}_{M-1}+\boldsymbol{w}_{M-1}^{T} \boldsymbol{U} \boldsymbol{w}_{M-1} \\
+\left(\boldsymbol{A} \boldsymbol{x}_{M-1}+\boldsymbol{B} \boldsymbol{u}_{M-1}+\right. \\
\left.\boldsymbol{F} \boldsymbol{w}_{M-1}\right)^{T} \boldsymbol{P}_{M}\left(\boldsymbol{A} \boldsymbol{x}_{M-1}\right. \\
\left.+\boldsymbol{B} \boldsymbol{u}_{M-1}+\boldsymbol{F} \boldsymbol{w}_{M-1}\right)
\end{array}
$$


The partial derivative of $\boldsymbol{\Gamma}_{M-1}^{\prime}$ in (20) w.r.t. $\boldsymbol{u}_{M-1}$ comes as:

$$
\begin{gathered}
\partial \boldsymbol{\Gamma}_{M-1}^{\prime} / \partial \boldsymbol{u}_{M-1}=2\left[\boldsymbol{R} \boldsymbol{u}_{M-1}\right. \\
\left.+\boldsymbol{B}^{T} \boldsymbol{P}_{M}\left(\boldsymbol{A} \boldsymbol{x}_{M-1}+\boldsymbol{B} \boldsymbol{u}_{M-1}+\boldsymbol{F} \boldsymbol{w}_{M-1}\right)\right] \\
\because \partial \boldsymbol{\Gamma}_{M-1}^{\prime} / \partial \boldsymbol{u}_{M-1}=\mathbf{0}, \text { for } \boldsymbol{u}_{M-1}=\boldsymbol{u}_{M-1}^{o p t} \\
\therefore \boldsymbol{R} \boldsymbol{u}_{M-1}^{o p t}+\boldsymbol{B}^{T} \boldsymbol{P}_{M}\left(\boldsymbol{A} \boldsymbol{x}_{M-1}+\boldsymbol{B} \boldsymbol{u}_{M-1}^{o p t}+\boldsymbol{F} \boldsymbol{w}_{M-1}\right)=\mathbf{0} \\
\Rightarrow \boldsymbol{u}_{M-1}^{o p t}=-\left(\boldsymbol{G}_{M-1} \boldsymbol{x}_{M-1}+\boldsymbol{Z}_{M-1} \boldsymbol{w}_{M-1}\right)
\end{gathered}
$$

where, $\boldsymbol{G}_{M-1}=\left(\boldsymbol{R}+\boldsymbol{B}^{T} \boldsymbol{P}_{M} \boldsymbol{B}\right)^{-1} \boldsymbol{B}^{T} \boldsymbol{P}_{M} \boldsymbol{A}$,

$$
\boldsymbol{Z}_{M-1}=\left(\boldsymbol{R}+\boldsymbol{B}^{T} \boldsymbol{P}_{M} \boldsymbol{B}\right)^{-1} \boldsymbol{B}^{T} \boldsymbol{P}_{M} \boldsymbol{F}
$$

Also, since $\partial^{2} \boldsymbol{\Gamma}_{M-1}^{\prime} /\left(\partial \boldsymbol{u}_{M-1}\right)^{2}=\left(\boldsymbol{R}+\boldsymbol{B}^{T} \boldsymbol{P}_{M} \boldsymbol{B}\right)>0$ (because $\boldsymbol{R}>0$ and $\boldsymbol{P}_{M} \geq 0$ ), and $\boldsymbol{\Gamma}_{M-1}^{\prime}$ is a quadratic function of $\boldsymbol{u}_{M-1}$, hence, $\boldsymbol{u}_{M-1}^{o p t}$ provides the global minimum value for $\boldsymbol{\Gamma}_{M-1}^{\prime}$. Substituting $\boldsymbol{u}_{M-1}^{o p t}$ from (24) for $\boldsymbol{u}_{M-1}$ in (19):

$$
\begin{aligned}
\boldsymbol{\Gamma}_{M-1}^{\prime o p t}=\boldsymbol{x}_{M-1}^{T} \boldsymbol{P}_{M-1} \boldsymbol{x}_{M-1}+ & 2 \boldsymbol{x}_{M-1}^{T} \boldsymbol{V}_{M-1} \boldsymbol{w}_{M-1} \\
& +\boldsymbol{w}_{M-1}^{T} \boldsymbol{W}_{M-1} \boldsymbol{w}_{M-1}
\end{aligned}
$$

where, $\boldsymbol{P}_{M-1}=\boldsymbol{Q}+\boldsymbol{G}_{M-1}^{T} \boldsymbol{R G}_{M-1}$

$$
+\left(\boldsymbol{A}-\boldsymbol{B G}_{M-1}\right)^{T} \boldsymbol{P}_{M}\left(\boldsymbol{A}-\boldsymbol{B} \boldsymbol{G}_{M-1}\right),
$$

$$
\boldsymbol{V}_{M-1}=\boldsymbol{G}_{M-1}^{T} \boldsymbol{R} \boldsymbol{Z}_{M-1}
$$$$
+\left(\boldsymbol{A}-\boldsymbol{B} \boldsymbol{G}_{M-1}\right)^{T} \boldsymbol{P}_{M}\left(\boldsymbol{F}-\boldsymbol{B} \boldsymbol{Z}_{M-1}\right),
$$

$$
\begin{aligned}
\boldsymbol{W}_{M-1}=\boldsymbol{U} & +\boldsymbol{Z}_{M-1}^{T} \boldsymbol{R} \boldsymbol{Z}_{M-1} \\
& +\left(\boldsymbol{F}-\boldsymbol{B} \boldsymbol{Z}_{M-1}\right)^{T} \boldsymbol{P}_{M}\left(\boldsymbol{F}-\boldsymbol{B} \boldsymbol{Z}_{M-1}\right)
\end{aligned}
$$

The net cost for $k=(M-2),(M-1)$ and $M$, such that the net cost for $k=(M-1)$ and $M$ is optimal (given by $\Gamma_{M-1}^{\prime o p t}$ ), is $\boldsymbol{\Gamma}_{M-2}^{\prime}=\boldsymbol{x}_{M-2}^{T} \boldsymbol{Q} \boldsymbol{x}_{M-2}+\boldsymbol{u}_{M-2}^{T} \boldsymbol{R} \boldsymbol{u}_{M-2}+\boldsymbol{w}_{M-2}^{T} \boldsymbol{U} \boldsymbol{w}_{M-2}+$ $\Gamma_{M-1}^{\prime o p t}$, and applying the same steps which are used to find $\Gamma_{M-1}^{\prime o p t}$, the expressions for $\boldsymbol{u}_{M-2}^{o p t}$ and $\boldsymbol{\Gamma}_{M-2}^{\prime o p t}$ come as follows.

$$
\boldsymbol{u}_{M-2}^{o p t}=-\left(\boldsymbol{G}_{M-2} \boldsymbol{x}_{M-2}+\boldsymbol{Z}_{M-2} \boldsymbol{w}_{M-2}\right),
$$

where, $\boldsymbol{G}_{M-2}=\left(\boldsymbol{R}+\boldsymbol{B}^{T} \boldsymbol{P}_{M-1} \boldsymbol{B}\right)^{-1} \boldsymbol{B}^{T} \boldsymbol{P}_{M-1} \boldsymbol{A}$,

$$
\begin{aligned}
\boldsymbol{Z}_{M-2}=\left(\boldsymbol{R}+\boldsymbol{B}^{T} \boldsymbol{P}_{M-1} \boldsymbol{B}\right)^{-1} \boldsymbol{B}^{T}\left(\boldsymbol{P}_{M-1} \boldsymbol{F}\right. & \left.+\boldsymbol{V}_{M-1} \boldsymbol{J}_{M-1}\right) ; \\
\boldsymbol{\Gamma}_{M-2}^{\prime o p t}=\boldsymbol{x}_{M-2}^{T} \boldsymbol{P}_{M-2} \boldsymbol{x}_{M-2}+ & 2 \boldsymbol{x}_{M-2}^{T} \boldsymbol{V}_{M-2} \boldsymbol{w}_{M-2} \\
+ & \boldsymbol{w}_{M-2}^{T} \boldsymbol{W}_{M-2} \boldsymbol{w}_{M-2},
\end{aligned}
$$

where, $\boldsymbol{P}_{M-2}=\left(\boldsymbol{A}-\boldsymbol{B G}_{M-2}\right)^{T} \boldsymbol{P}_{M-1}\left(\boldsymbol{A}-\boldsymbol{B}_{\boldsymbol{G}_{M-2}}\right)$

$$
+\boldsymbol{G}_{M-2}^{T} \boldsymbol{R} \boldsymbol{G}_{M-2}+\boldsymbol{Q},
$$

$\boldsymbol{V}_{M-2}=\left(\boldsymbol{A}-\boldsymbol{B} \boldsymbol{G}_{M-2}\right)^{T} \boldsymbol{P}_{M-1}\left(\boldsymbol{F}-\boldsymbol{B} \boldsymbol{Z}_{M-2}\right)$

$$
+\left(\boldsymbol{A}-\boldsymbol{B} \boldsymbol{G}_{M-2}\right)^{T} \boldsymbol{V}_{M-1} \boldsymbol{J}_{M-1}+\boldsymbol{G}_{M-2}^{T} \boldsymbol{R} \boldsymbol{Z}_{M-2},
$$$$
\boldsymbol{W}_{M-2}=\left(\boldsymbol{F}-\boldsymbol{B} \boldsymbol{Z}_{M-2}\right)^{T}\left[\boldsymbol{P}_{M-1}\left(\boldsymbol{F}-\boldsymbol{B} \boldsymbol{Z}_{M-2}\right)\right.
$$$$
\left.+\boldsymbol{V}_{M-1} \boldsymbol{J}_{M-1}\right]+\boldsymbol{J}_{M-1}^{T} \boldsymbol{W}_{M-1} \boldsymbol{J}_{M-1}
$$$$
+\boldsymbol{Z}_{M-2}^{T} \boldsymbol{R} \boldsymbol{Z}_{M-2}+\boldsymbol{U}
$$

After evaluating the terms $\boldsymbol{u}_{M-3}^{o p t}$ and $\boldsymbol{\Gamma}_{M-3}^{o p t}$ following the similar steps as above, their expressions are found to be similar to (31) and (34), respectively, the only difference being that $M-2$ gets replaced by $M-3$, and $M-1$ gets replaced by $M-2$. This similarity holds for other expressions of $\boldsymbol{u}_{k}^{o p t}$ and $\boldsymbol{\Gamma}_{k}^{\prime o p t}$, for all $k<M-3$. Thus, applying induction for $k<M$ and using initial conditions $\boldsymbol{V}_{M}=\mathbf{0}_{m \times(n+1)}$ and $\boldsymbol{P}_{M}=\boldsymbol{Q}$, the optimal cost for $\Gamma^{\prime}$ in (16) is found to be $\Gamma_{0}^{\prime o p t}$ (and is evaluated by iteratively finding the sequence $\Gamma_{M}^{\prime o p t}, \Gamma_{M-1}^{\prime o p t}$, $\ldots, \Gamma_{1}^{o p t}, \Gamma_{0}^{\prime o p t}$ ) and the corresponding optimal control is found to be:

$$
\boldsymbol{u}_{k}^{o p t}=-\left(\boldsymbol{G}_{k} \boldsymbol{x}_{k}+\boldsymbol{Z}_{k} \boldsymbol{w}_{k}\right), 0 \leq k<M
$$

where, $\boldsymbol{G}_{k}=\left(\boldsymbol{R}+\boldsymbol{B}^{T} \boldsymbol{P}_{k+1} \boldsymbol{B}\right)^{-1} \boldsymbol{B}^{T} \boldsymbol{P}_{k+1} \boldsymbol{A}$

$$
\begin{array}{r}
\boldsymbol{Z}_{k}=\left(\boldsymbol{R}+\boldsymbol{B}^{T} \boldsymbol{P}_{k+1} \boldsymbol{B}\right)^{-1} \boldsymbol{B}^{T}\left(\boldsymbol{P}_{k+1} \boldsymbol{F}+\boldsymbol{V}_{k+1} \boldsymbol{J}_{k+1}\right) \\
\boldsymbol{P}_{k}=\boldsymbol{Q}+\boldsymbol{G}_{k}^{T} \boldsymbol{R} \boldsymbol{G}_{k}+\left(\boldsymbol{A}-\boldsymbol{B} \boldsymbol{G}_{k}\right)^{T} \boldsymbol{P}_{k+1}\left(\boldsymbol{A}-\boldsymbol{B}_{k}\right) \\
\boldsymbol{V}_{k}=\boldsymbol{G}_{k}^{T} \boldsymbol{R} \boldsymbol{Z}_{k}+\left(\boldsymbol{A}-\boldsymbol{B} \boldsymbol{G}_{k}\right)^{T}\left[\boldsymbol{P}_{k+1}\left(\boldsymbol{F}-\boldsymbol{B} \boldsymbol{Z}_{k}\right)\right. \\
\left.+\boldsymbol{V}_{k+1} \boldsymbol{J}_{k+1}\right]
\end{array}
$$

From above expressions it may be inferred that $\boldsymbol{u}_{k}^{o p t}$ is independent of $\boldsymbol{W}_{k}$. Moreover, $\boldsymbol{P}_{k}$ can be stated as follows (using (41)).

$$
\begin{gathered}
\boldsymbol{P}_{k}=\boldsymbol{Q}+\boldsymbol{G}_{k}^{T}\left(\boldsymbol{R}+\boldsymbol{B}^{T} \boldsymbol{P}_{k+1} \boldsymbol{B}\right) \boldsymbol{G}_{k}-\boldsymbol{G}_{k}^{T} \boldsymbol{B}^{T} \boldsymbol{P}_{k+1} \boldsymbol{A} \\
+\boldsymbol{A}^{T} \boldsymbol{P}_{k+1}\left(\boldsymbol{A}-\boldsymbol{B} \boldsymbol{G}_{k}\right) \\
\because \boldsymbol{G}_{k}^{T}\left(\boldsymbol{R}+\boldsymbol{B}^{T} \boldsymbol{P}_{k+1} \boldsymbol{B}\right) \boldsymbol{G}_{k}=\boldsymbol{G}_{k}^{T} \boldsymbol{B}^{T} \boldsymbol{P}_{k+1} \boldsymbol{A}(\text { from (39)) } \\
\therefore \boldsymbol{P}_{k}=\boldsymbol{Q}+\boldsymbol{A}^{T} \boldsymbol{P}_{k+1}\left(\boldsymbol{A}-\boldsymbol{B} \boldsymbol{G}_{k}\right),
\end{gathered}
$$

After substituting $\boldsymbol{G}_{k}$ from (39) in (44), the following expression for $\boldsymbol{P}_{k}$ is found.

$$
\boldsymbol{P}_{k}=\boldsymbol{Q}+\boldsymbol{A}^{T}\left(\boldsymbol{P}_{k+1} \boldsymbol{B}\left(\boldsymbol{R}+\boldsymbol{B}^{T} \boldsymbol{P}_{k+1} \boldsymbol{B}\right)^{-1} \boldsymbol{B}^{T} \boldsymbol{P}_{k+1}\right) \boldsymbol{A}
$$

In a similar way, $\boldsymbol{V}_{k}$ (using (42)) can be re-expressed as:

$$
\begin{gathered}
\boldsymbol{V}_{k}=\left(\boldsymbol{A}-\boldsymbol{B} \boldsymbol{G}_{k}\right)^{T}\left(\boldsymbol{P}_{k+1} \boldsymbol{F}+\boldsymbol{V}_{k+1} \boldsymbol{J}_{k+1}\right) \\
\quad+\boldsymbol{G}_{k}^{T}\left(\boldsymbol{R}+\boldsymbol{B}^{T} \boldsymbol{P}_{k+1} \boldsymbol{B}\right) \boldsymbol{Z}_{k}-\boldsymbol{A}^{T} \boldsymbol{P}_{k+1} \boldsymbol{B} \boldsymbol{Z}_{k} \\
\because \boldsymbol{G}_{k}^{T}\left(\boldsymbol{R}+\boldsymbol{B}^{T} \boldsymbol{P}_{k+1} \boldsymbol{B}\right) \boldsymbol{Z}_{k}=\boldsymbol{A}^{T} \boldsymbol{P}_{k+1} \boldsymbol{B} \boldsymbol{Z}_{k}(\text { using }(39)) \\
\therefore \boldsymbol{V}_{k}=\left(\boldsymbol{A}-\boldsymbol{B} \boldsymbol{G}_{k}\right)^{T}\left(\boldsymbol{P}_{k+1} \boldsymbol{F}+\boldsymbol{V}_{k+1} \boldsymbol{J}_{k+1}\right)
\end{gathered}
$$

From (44):

$$
\left(\boldsymbol{A}-\boldsymbol{B G}_{k}\right)^{T}=\left(\boldsymbol{P}_{k}-\boldsymbol{Q}\right) \boldsymbol{A}^{-1} \boldsymbol{P}_{k+1}^{-1}
$$

Substituting $\left(\boldsymbol{A}-\boldsymbol{B G}_{k}\right)^{T}$ from (48) in (47):

$$
\boldsymbol{V}_{k}=\left(\boldsymbol{P}_{k}-\boldsymbol{Q}\right) \boldsymbol{A}^{-1}\left(\boldsymbol{F}+\boldsymbol{P}_{k+1}^{-1} \boldsymbol{V}_{k+1} \boldsymbol{J}_{k+1}\right)
$$

Using (39), $\boldsymbol{Z}_{k}$ in (40) can be stated as:

$$
\begin{gathered}
\boldsymbol{Z}_{k}=\boldsymbol{G}_{k} \boldsymbol{A}^{-1}\left(\boldsymbol{F}+\boldsymbol{P}_{k+1}^{-1} \boldsymbol{V}_{k+1} \boldsymbol{J}_{k+1}\right) ; \text { and using (49), } \\
\Rightarrow \boldsymbol{Z}_{k}=\boldsymbol{G}_{k}\left(\boldsymbol{P}_{k}-\boldsymbol{Q}\right)^{-1} \boldsymbol{V}_{k}
\end{gathered}
$$


Partitioning $\boldsymbol{V}_{k}$ in (47) as $\left[\begin{array}{ll}\boldsymbol{L}_{k} & \boldsymbol{L}_{k}^{\prime}\end{array}\right], \boldsymbol{L}_{k} \in \mathbb{R}^{m \times n}, \boldsymbol{L}_{k}^{\prime} \in$ $\mathbb{R}^{m \times 1}$ :

$$
\begin{gathered}
{\left[\begin{array}{ll}
\boldsymbol{L}_{k} & \boldsymbol{L}_{k}^{\prime}
\end{array}\right]=\left(\boldsymbol{A}-\boldsymbol{B} \boldsymbol{G}_{k}\right)^{T}\left(\boldsymbol{P}_{k+1} \boldsymbol{F}+\left[\begin{array}{ll}
\boldsymbol{L}_{k+1} & \boldsymbol{L}_{k+1}^{\prime}
\end{array}\right] \boldsymbol{J}_{k+1}\right)} \\
\Rightarrow\left[\begin{array}{ll}
\boldsymbol{L}_{k} & \boldsymbol{L}_{k}^{\prime}
\end{array}\right]=\left(\boldsymbol{A}-\boldsymbol{B} \boldsymbol{G}_{k}\right)^{T}\left(\boldsymbol{P}_{k+1}\left[\begin{array}{ll}
\boldsymbol{E} & \mathbf{0}_{m \times 1}
\end{array}\right]\right. \\
\left.+\left[\begin{array}{ll}
\boldsymbol{L}_{k+1} & \boldsymbol{L}_{k+1}^{\prime}
\end{array}\right]\left[\begin{array}{cc}
\boldsymbol{I}_{n} & \boldsymbol{u}_{k+1}^{\prime}-\boldsymbol{u}_{k}^{\prime} \\
\mathbf{0}_{1 \times n} & 1
\end{array}\right]\right) \\
\Rightarrow \boldsymbol{L}_{k}=\left(\boldsymbol{A}-\boldsymbol{B} \boldsymbol{G}_{k}\right)^{T}\left(\boldsymbol{P}_{k+1} \boldsymbol{E}+\boldsymbol{L}_{k+1}\right), \text { and } \\
\boldsymbol{L}_{k}^{\prime}=\left(\boldsymbol{A}-\boldsymbol{B} \boldsymbol{G}_{k}\right)^{T}\left(\boldsymbol{L}_{k+1}\left(\boldsymbol{u}_{k+1}^{\prime}-\boldsymbol{u}_{k}^{\prime}\right)+\boldsymbol{L}_{k+1}^{\prime}\right)
\end{gathered}
$$

Partitioning $\boldsymbol{Z}_{k}$ in (38) as $\left[\begin{array}{ll}\boldsymbol{K}_{k} & \boldsymbol{K}_{k}^{\prime}\end{array}\right], \boldsymbol{K}_{k} \in \mathbb{R}^{l \times n}, \boldsymbol{K}_{k}^{\prime} \in$ $\mathbb{R}^{l \times 1}$, where $l$ is the number of elements in $\boldsymbol{u}_{k}$ :

$$
\begin{gathered}
\boldsymbol{u}_{k}^{o p t}=-\left(\boldsymbol{G}_{k} \boldsymbol{x}_{k}+\left[\begin{array}{ll}
\boldsymbol{K}_{k} & \boldsymbol{K}_{k}^{\prime}
\end{array}\right]\left[\begin{array}{c}
\boldsymbol{u}_{k}^{\prime} \\
1
\end{array}\right]\right), \\
\Rightarrow \boldsymbol{u}_{k}^{o p t}=-\left(\boldsymbol{G}_{k} \boldsymbol{x}_{k}+\boldsymbol{K}_{k} \boldsymbol{u}_{k}^{\prime}+\boldsymbol{K}_{k}^{\prime}\right)
\end{gathered}
$$

and using (50), $\left[\begin{array}{ll}\boldsymbol{K}_{k} & \boldsymbol{K}_{k}^{\prime}\end{array}\right]=\boldsymbol{G}_{k}\left(\boldsymbol{P}_{k}-\boldsymbol{Q}\right)^{-1}\left[\begin{array}{ll}\boldsymbol{L}_{k} & \boldsymbol{L}_{k}^{\prime}\end{array}\right] \Rightarrow$

$$
\boldsymbol{K}_{k}=\boldsymbol{G}_{k}\left(\boldsymbol{P}_{k}-\boldsymbol{Q}\right)^{-1} \boldsymbol{L}_{k} ; \boldsymbol{K}_{k}^{\prime}=\boldsymbol{G}_{k}\left(\boldsymbol{P}_{k}-\boldsymbol{Q}\right)^{-1} \boldsymbol{L}_{k}^{\prime}
$$

Hence, with (39), (45), (52)-(55), Theorem 1 has been proved.

The above optimal solution has been termed as the "extended linear quadratic regulator (ELQR)'. Finite horizon case can be applied to the ELQR solution only if the sequence of external disturbances is known to be finite, otherwise only infinite horizon case is applicable. Provided $(\boldsymbol{A}, \boldsymbol{B})$ is stabilizable, the solutions for the infinite horizon case for $\boldsymbol{P}_{k}$, $\boldsymbol{G}_{k}, \boldsymbol{K}_{k}$ and $\boldsymbol{L}_{k}$ exist, and the solutions are $\boldsymbol{G}, \boldsymbol{P}$ (given by (6)-(7)), and $L, K$ (given by (56)-(57)).

$$
\boldsymbol{L}=(\boldsymbol{A}-\boldsymbol{B} \boldsymbol{G})^{T}(\boldsymbol{P} \boldsymbol{E}+\boldsymbol{L})=(\boldsymbol{P}-\boldsymbol{Q}) \boldsymbol{A}^{-1}\left(\boldsymbol{E}+\boldsymbol{P}^{-1} \boldsymbol{L}\right),
$$

(this is because $(\boldsymbol{A}-\boldsymbol{B} \boldsymbol{G})^{T}=(\boldsymbol{P}-\boldsymbol{Q}) \boldsymbol{A}^{-1} \boldsymbol{P}^{-1}$ from (48))

$$
\begin{aligned}
& \Rightarrow \boldsymbol{L}=\left(\boldsymbol{A}(\boldsymbol{P}-\boldsymbol{Q})^{-1}-\boldsymbol{P}^{-1}\right)^{-1} \boldsymbol{E} \\
\boldsymbol{K}= & \boldsymbol{G}(\boldsymbol{P}-\boldsymbol{Q})^{-1} \boldsymbol{L}, \text { substituting } \boldsymbol{L} \text { from }(56): \\
& \Rightarrow \boldsymbol{K}=\boldsymbol{G}\left(\boldsymbol{A}-\boldsymbol{P}^{-1}(\boldsymbol{P}-\boldsymbol{Q})\right)^{-1} \boldsymbol{E}
\end{aligned}
$$

The terms $\boldsymbol{G}_{k}$ and $\boldsymbol{P}_{k}$ for ELQR remain same as LQR. The terms $\boldsymbol{K}_{k}$ and $\boldsymbol{L}_{k}$ do not depend on $\boldsymbol{u}_{k}^{\prime}$, and hence can be calculated just with the knowledge of $A, B, E, Q$ and $R$. The terms $\boldsymbol{K}_{k}^{\prime}$ and $\boldsymbol{L}_{k}^{\prime}$ need the knowledge of the present and future time samples of $\boldsymbol{u}_{k}^{\prime}$. Therefore, it is assumed that $\boldsymbol{u}_{k}^{\prime}$ is known in advance, and if the sequence of $\boldsymbol{u}_{k}^{\prime}$ is not known in advance, the terms which can be accurately calculated are $\boldsymbol{G}_{k}, \boldsymbol{P}_{k}, \boldsymbol{K}_{k}$ and $\boldsymbol{L}_{k}$, whereas the terms $\boldsymbol{K}_{k}^{\prime}$ and $\boldsymbol{L}_{k}^{\prime}$ can only be estimated/predicted using the estimated/predicted values of $\boldsymbol{u}_{k}^{\prime}$.

\section{Application example: Decentralized control OF POWER SYSTEM DYNAMICS}

ELQR can be employed for control of any system which can be expressed in the form of (8). In the following illustrative example a highly non-linear power system model is controlled using the ELQR methodology.

\section{A. Power system modeling}

It was proved and demonstrated in [6] that if the measurements of voltage magnitude and voltage phase acquired from the terminal bus of a synchronous generation unit in a multimachine power system are treated as external disturbances, the dynamic equations for that unit get decoupled from rest of the power system. The equation for the $i^{\text {th }}$ unit in the system is written in the decentralized form as (58):

$$
\dot{\boldsymbol{x}}_{c i}(t)=\boldsymbol{g}_{i}\left(\boldsymbol{x}_{c i}(t), \boldsymbol{u}_{c i}(t), \boldsymbol{u}_{c i}^{\prime}(t)\right)
$$

The subscript $c$ denotes that (58) is in continuous-time form. $\boldsymbol{g}_{i}$ denotes the differential function of the various states, $\boldsymbol{x}_{c i}$, the external disturbances, $\boldsymbol{u}_{c i}^{\prime}$ (which are the voltage magnitude and phase), and the control input $\boldsymbol{u}_{c i}$ (which is the input to the automatic voltage regulator). The details of $\boldsymbol{g}_{i}$ and the various states of a machine are given in [10].

A power system can be controlled in a decentralized manner using the decoupled equations given by (58). Additionally, nonlinear Kalman filtering can generate dynamic state estimates for that unit with accuracy and precision. This algorithm of decentralized dynamic state estimation (DSE) is employed for getting the estimates of states needed by the ELQR [6].

\section{B. Damping control}

An operating constraint for power systems is that the damping ratios of all the closed-loop electromechanical eigenvalues of the system should be more than a specified percentage. Thus, the electromechanical eigenvalues for a generation unit should be in the continuous-plane's left half, inside a conical section. This conical section corresponds to a spiral section [1] in the discrete-plane, and thus, the discrete-domain eigenvalues must be inside this spiral. Since enclosing the closed-loop poles inside a spiral is mathematically very difficult, the spiral is substituted with a circle, and the eigenvalues are enclosed in that circle. A circle best substitutes the spiral if the spiral is tangentially intersected by the circle at the coordinates at which the electromechanical eigenvalues are supposed to lie. As each machine has only one pair of electromechanical poles, a unique substituting disk can be found for this pair.

It can be shown from this paper's Theorem 1 and Theorem 2 in [12] that the control law for enclosing a system's closedloop eigenvalues inside a circle of radius $a$ and center $(b, 0)$ is similar to (6)-(7) and (56)-(57), the only difference is that $\boldsymbol{A}, \boldsymbol{B}$ and $\boldsymbol{E}$ are replaced by $(\boldsymbol{A}-b \boldsymbol{I}) / a, \boldsymbol{B} / a$ and $\boldsymbol{E} / a$, respectively.

\section{ELQR application}

The continuous-time equation in (58) is linearized and discretized to covert it in the form of equation (8) so that the ELQR control gains can be calculated (after modifying the state matrices according to Section IV-B). This linearization and discretization of (58) is done every second, so that the non-linearity in (58) is dynamically reflected in the control gains. It should be noted that as the voltage magnitude and phase can only be known for past and present samples, the term $\boldsymbol{K}_{k}^{\prime}$ cannot be calculated. Also, offline values of the 
external disturbances showed that $\boldsymbol{K}_{k}^{\prime}$ provides insignificant contribution to the optimal control law of Theorem 1. Hence, only the gains $\boldsymbol{G}$ and $\boldsymbol{K}$ are used for control.

\section{Implementation and validation}

The study of decentralized control of power systems using the ELQR methodology has been conducted on a 16-machine, 68-bus benchmark system. The system and its parameters are described in detail in [10].

MATLAB Simulink has been used for simulating the system and the ELQR control is applied on each machine in the system. Comparison simulations are also conducted by replacing the ELQR with a classical PSS on each machine [11].

\section{E. Control performance}

At the start of the simulation the power system is in steady state. At $t=1 \mathrm{~s}$ a 3 -phase fault is simulated on one of the links in the power flow path between two nodes. The link is immediately disconnected to clear the fault.

Fig. 1 plots the relative speed of the rotors of units 13 and 16 and the electric-power flow in the link between nodes 60 and 61 (which is a link between two areas) for PSS control versus ELQR control. Table I presents a comparison of total costs for three operating conditions. It can be observed that the net quadratic costs decrease by $24 \%$ (on an average) for the case of ELQR when compared to PSS.
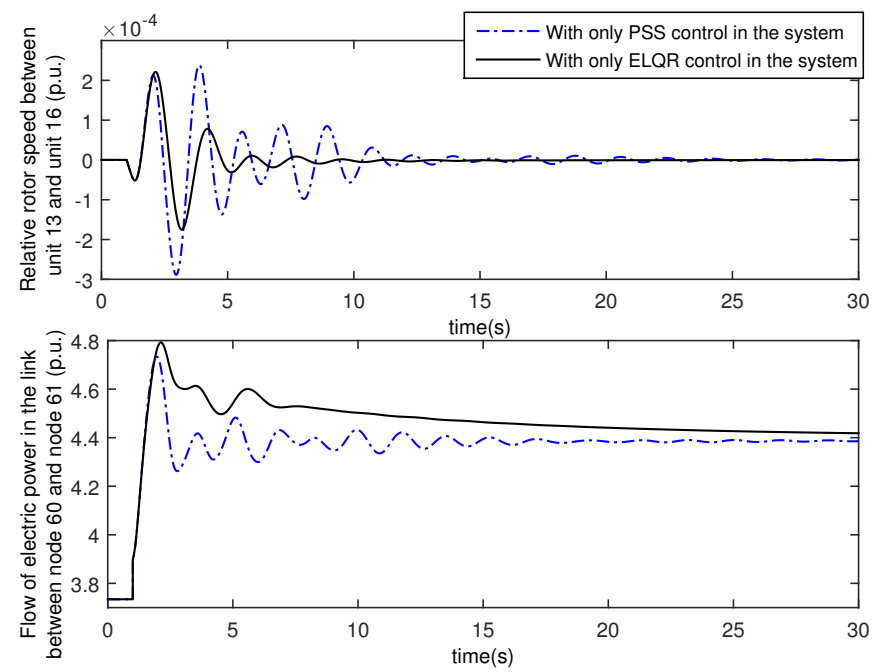

Fig. 1. Control performance of PSS vs ELQR

TABLE I

PSS VS ELQR: QUADRATIC COSTS

\begin{tabular}{|c|c|c|}
\hline $\begin{array}{c}\text { Operating condition: } \\
\text { faulted link and inter-area } \\
\text { electric-power flow }\end{array}$ & $\begin{array}{c}\text { Net quadratic } \\
\text { costs for } \\
\text { PSS control (p.u.) }\end{array}$ & $\begin{array}{c}\text { Net quadratic } \\
\text { costs for } \\
\text { ELQR control (p.u.) }\end{array}$ \\
\hline $53-54,700 \mathrm{MW}$ & 1.87 & 1.40 \\
$53-54,100 \mathrm{MW}$ & 0.30 & 0.18 \\
$27-53,900 \mathrm{MW}$ & 0.20 & 0.18 \\
\hline
\end{tabular}

\section{CONCLUSIONS}

An optimal control scheme has been proposed for systems in which both control inputs and external disturbances are present. The scheme is termed as extended LQR, and its cost effectiveness has been rigorously derived. The applicability of the scheme has been shown on the model of a complex multimachine system.

\section{REFERENCES}

[1] Charles L. Philips and H. Troy Nagel, Digital Control System Analysis and Design. 3rd ed. U.S.A.: Prentice Hall, 1994.

[2] R. E. Kalman, "A new approach to linear filtering and prediction problems," Transactions of the ASME, Journal of Basic Engineering, vol. 82, pp. 34-45, 1960.

[3] Jingqing Han, "From PID to Active Disturbance Rejection Control," IEEE Transactions on Industrial Electronics, vol. 56, no. 3, pp. 900906, Mar. 2009.

[4] Sung-Han Son; Kang-Bak Park, "Discretization behaviors of equivalent control based sliding-mode control systems with external disturbances," 30th Annual Conference of IEEE in Industrial Electronics Society, 2004. IECON 2004., vol.3, pp. 2264-2268 vol. 3, 2-6 Nov. 2004.

[5] Zhiqiang Gao, "Active disturbance rejection control: a paradigm shift in feedback control system design," American Control Conference, 2006, 14-16 Jun. 2006.

[6] A. K. Singh; B. C. Pal, "Decentralized Dynamic State Estimation in Power Systems Using Unscented Transformation," IEEE Transactions on Power Systems, vol. 29, no. 2, pp. 794-804, Mar. 2014.

[7] C. D. Johnson, "Accommodation of external disturbances in linear regulator and servomechanism problems," IEEE Transactions on Automatic Control, vol. 16, no. 6, pp. 635-644, Dec. 1971.

[8] Eric Ostertag, "Deterministic Disturbances Compensation. Disturbance Observer," in Mono-and Multivariable Control and Estimation: Linear, Quadratic and LMI Methods vol. 2, Berlin: Springer Science \& Business Media, 2011.

[9] C. D. Johnson, "Accommodation of disturbances in optimal control problems" International Journal of Control vol. 15, no. 2, pp. 209-231, 1972.

[10] B. Pal; B. Chaudhuri, Robust Control in Power Systems. New York, U.S.A.: Springer, 2005

[11] E. V. Larsen and D. A. Swann, "Applying Power System Stabilizers Parts I, II and III," IEEE Transactions on Power Apparatus and Systems, vol. PAS-100, no. 6, pp. 3017-3046, 1981.

[12] K. Furuta, S. Kim, "Pole assignment in a specified disk," IEEE Trans. Autom. Control, vol. 32, no. 5, pp. 423-427, May 1987.

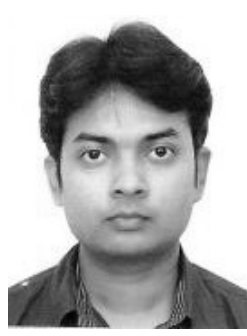

Abhinav K. Singh (S'12-M'15) received B.Tech. degree from Indian Institute of Technology, New Delhi, India, and Ph.D. degree from Imperial College London, London, U.K., in 2010 and 2015, respectively, all in electrical engineering.

Currently, he is working as a Research Associate at Imperial College London. His current research interests include decentralized estimation and control, networked control and communication aspects of power systems.

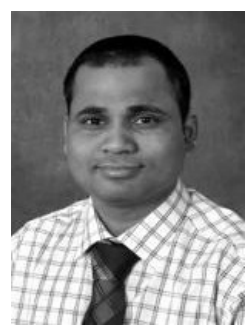

Bikash C. Pal (M'00-SM'02-F'13) received B.E.E. (with honors) degree from Jadavpur University, Calcutta, India, M.E. degree from the Indian Institute of Science, Bangalore, India, and Ph.D. degree from Imperial College London, London, U.K., in 1990, 1992, and 1999, respectively, all in electrical engineering.

Currently, he is a Professor in the Department of Electrical and Electronic Engineering, Imperial College London. $\mathrm{He}$ is Editor-in-Chief of IEEE Transactions on Sustainable Energy and Fellow of IEEE for his contribution to power system stability and control. His current research interests include state estimation, and power system dynamics. 